\section{RXRA DT448/9PP generates a dominant active variant capable of inducing maturation in acute myeloid leukemia cells}

\author{
Orsola di Martino,,$^{1^{\star}}$ Margaret A. Ferris, ${ }^{2 \star}$ Gayla Hadwiger, ${ }^{1}$ Soyi Sarkar, ${ }^{1}$ \\ Anh Vu, ${ }^{1}$ María P. Menéndez-Gutiérrez, ${ }^{3}$ Mercedes Ricote ${ }^{3}$ and John S. Welch
}

${ }^{1}$ Department of Internal Medicine, Washington University, St Louis, MO, USA; ${ }^{2}$ Department of Pediatrics, Washington University, St Louis, MO, USA and ${ }^{3}$ Myocardial Pathophysiology Area, Centro Nacional de Investigaciones Cardiovasculares (CNIC), Madrid, Spain

*OdM and MAF contributed equally as co-first authors.

\section{ABSTRACT}

$\mathrm{R}$ ARA and RXRA contribute to myeloid maturation in both mice and humans, and deletion of Rxra and Rxrb augments leukemic growth in mice. While defining the domains of RXRA that are required for anti-leukemic effects in murine KMT2A-MLLT3 leukemia cells, we unexpectedly identified RXRA DT448/9PP as a constitutively active variant capable of inducing maturation and loss of their proliferative phenotype. RXRA DT448/9PP was associated with ligand-independent activity in reporter assays, with enhanced co-activator interactions, reduced engraftment in vivo, and activation of myeloid maturation transcriptional signatures that overlapped with those of cells treated with the potent RXRA agonist bexarotene, suggestive of constitutive activity that leads to leukemic maturation. Phenotypes of RXRA DT448/9PP appear to differ from those of two other RXRA mutations with forms of constitutive activity (F318A and S427F), in that DT448/9PP activity was resistant to mutations at critical ligand-interacting amino acids (R316A/L326A) and was resistant to pharmacological antagonists, suggesting it may be ligand-independent. These data provide further evidence that activated retinoid $\mathrm{X}$ receptors can regulate myeloid maturation and provide a novel constitutively active variant that may be germane for broader studies of retinoid $X$ receptors in other settings.

\section{Introduction}

Retinoid receptors are highly conserved transcription factors that direct hematopoietic self-renewal and differentiation. ${ }^{1,2}$ Retinoids (vitamin A metabolites) bind directly to retinoid receptors, converting the receptors from transcriptional repressors to transcriptional activators. There are six types of retinoid receptor (RARA, RARB, RARG, $R X R A, R X R B, R X R G)$ with distinct tissue expression and subtle differences in their binding and response to different ligands. The retinoid receptors $R A R A$ and $R X R A$ undergo remarkable upregulation during myeloid maturation in both mice and humans, whereas RXRG is not detected. . $^{-5}$

Retinoid treatments, both in vitro and in vivo, facilitate hematopoietic stem cell maturation and lineage commitment. ${ }^{1,2}$ Until now, the clinical application of retinoids in hematology has been restricted to acute promyelocytic leukemia (treated with all-trans retinoic acid, or tretinoin, a pan-RAR ligand) and cutaneous T-cell leukemia (treated with bexarotene, a pan-RXR ligand). However, recent studies have observed activity of all-trans retinoic acid when combined with chemotherapy in acute myeloid leukemias other than the promyelocytic form, and the RARA super-enhancer has emerged as a potential biomarker of retinoid sensitivity. ${ }^{6-10}$

To better understand the molecular determinants of anti-leukemic retinoid activity, we evaluated a series of RXRA truncations and mutations to determine which might rescue phenotypes observed in $R x r a / R x r b$-deficient leukemia cells. ${ }^{5}$ We unexpectedly identified $R X R A$ DT448/9PP as a constitutively active variant capable of inducing maturation and loss of proliferative capacity in leukemia cells. In this study, we characterized the activity of this variant using cell culture assays, transcription reporter assays,
Haematologica 2022

Volume 107(2):417-426

\section{Correspondence:}

JOHN S. WELCH

jwelch@wustl.edu

Received: February 17, 2021.

Accepted: May 7, 2021.

Pre-published: June 17, 2021.

https://doi.org/10.3324/haematol.2021.278603

(C)2022 Ferrata Storti Foundation

Material published in Haematologica is covered by copyright. All rights are reserved to the Ferrata Storti Foundation. Use of published material is allowed under the following terms and conditions:

https://creativecommons.org/licenses/by-nc/4.0/legalcode. Copies of published material are allowed for personal or internal use. Sharing published material for non-commercial purposes is subject to the following conditions: https://creativecommons.org/licenses/by-nc/4.0/legalcode, sect. 3. Reproducing and sharing published material for commercial purposes is not allowed without permission in writing from the publisher. 
in vivo engraftment, and RNA sequencing, and present evidence of ligand-independent loss of proliferative capacity, transcriptional signatures of myeloid maturation that overlap with signatures induced by the potent RXR ligand bexarotene, and inhibition of engraftment and leukemic expansion in vivo. These data again suggest that programs of leukemic cell growth and maturation may be susceptible to retinoids and provide a novel constitutively active tool for further delineating the function and activity of retinoid receptors.

\section{Methods}

\section{Mice}

Mice were maintained in a specific pathogen-free barrier facility with a $12 \mathrm{~h}$ light-dark cycle. Upon weaning, all mice were housed in groups of up to five mice of the same sex per cage. Food and a water bottle were provided in a recess of the metal wire lid at the top of the cage. Cages were changed once every week. Six- to tenweek-old mice (C57B1/6 background) were typically used for the experiments. Equal numbers of male and female mice were used; no gender biases were noted.

\section{Hematopoietic cell culture}

Murine bone marrow $\mathrm{Kit}^{+}$cells were isolated using an Automacs Pro (Miltenyl Biotec, San Diego, CA, USA) according to the manufacturer's protocol. Kit cells were plated in progenitor expansion medium (RPMI 1640 medium, 15\% fetal bovine serum, stem factor [50 ng/mL], interleukin 3 [10 ng/mL], Flt3L [25 ng/mL], thrombopoietin $[10 \mathrm{ng} / \mathrm{mL}]$, L-glutamine $[2 \mathrm{mM}]$, sodium pyruvate $[1 \mathrm{mM}]$, HEPES buffer $[10 \mathrm{mM}]$, penicillin/streptomycin [100 units $/ \mathrm{mL}], \beta$-mercaptoethanol [50 $\mu \mathrm{M}]$ ) overnight and transduced with MSCV-KMT2A-MLLT3 retrovirus by spinfection with 10 $\mu \mathrm{g} / \mathrm{mL}$ polybrene and $10 \mathrm{mM}$ HEPES at $2400 \mathrm{rpm}, 30^{\circ} \mathrm{C}$ for $90 \mathrm{~min}$ in an Eppendorf 5810R centrifuge. Cells were transplanted into sublethally irradiated mice and subsequent leukemia harvested 4 to 6 months later. KMT2A-MLLT3 leukemia cells were cultured in vitro using similar media, but without Flt3L or thrombopoietin. KMT2A-MLLT3-RXR-knockout (KO) leukemia cells were derived

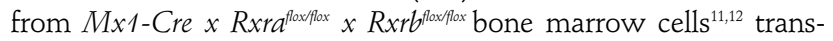
duced with MSCV-KMT2A-MLLT3 retrovirus and generated as described above. RXR deletion was induced by injecting $M x 1$-Cre

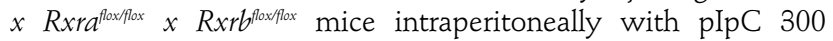
$\mu \mathrm{g} /$ mouse; four doses were given, every other day. $R X R$ deletion was confirmed by polymerase chain reaction analysis 4 weeks after mice had been treated with pIpC. THP1, and K562 cells were obtained from the American Type Culture Collection. Monomac6 and OCI-AML3 cells were obtained from the Deutsche Sammlung von Mikroorganismen und Zellkulturen.

\section{RNA sequencing}

KMT2A-MLLT3 leukemia cells were transduced with $M S C V$ RXRA-IRES-mCherry or MSCV-RXRA DT448/9PP-IRES-mCherry and treated with or without $250 \mathrm{nM}$ bexarotene. After $24 \mathrm{~h}$, $\mathrm{mCherry}^{+}$cells were sorted and total RNA was extracted with TRIzol LS (Ambion Life Technologies) and isolated on PureLink RNA Kit columns (Thermo Fisher Scientific). The quality of RNA was measured on a 2100 Bioanalyzer (Agilent). Sequencing libraries, each with individual Illumina indexes, were constructed using the TruSeq Stranded mRNA procedure (Sample Prep Kit v2; Illumina). Libraries were sequenced as paired-end $151 \mathrm{bp}$ reads on an Illumina NovaSeq instrument. Reads were aligned to the $\mathrm{mm} 10$ mouse reference genome, and transcription was quantified using kallisto version $0.43 .1^{13}$ and Ensembl transcripts version 95 . The $\mathrm{R}$ package edgeR was used to determine genes that were differentially expressed between groups: RXRA WT cells, RXRA WT cells treated with bexarotene, and RXRA DT448/9PP-transduced cells. Differentially expressed genes were defined as having an absolute $\log _{2}$ fold change $>2$ and a $P$-value $<0.0001$. Functional pathway analysis was performed using Panther Gene Ontology Analysis software ${ }^{14}$ with a Fisher exact test using the Bonferroni correction for multiple testing. Pathways were called if $P<0.05$ and fold enrichment was $>2$. Gene set enrichment analysis was performed using gene sets curated in the MSigDB database and GSEA 4.0.3 software; RRID:SCR_003199. ${ }^{15}$

\section{Study approval}

All animal procedures were approved by the Institutional Animal Care and Use Committee of Washington University.

\section{Results}

\section{Effects of RXRA DT448/9PP on maturation}

We recently evaluated a series of RXRA truncations and mutations to map the domains of RXRA required for antileukemic effects in murine KMT2A-MLLT3 (formerly MLLAF9) leukemia cells derived from $M x-C r e x$ Rxra $^{\text {fox } x \text { fox }} \times$ Rxr ffoxffox bone marrow cells (RXR-KO KMT2A-MLLT3 leukemia). ${ }^{5}$ Ghosh et al. had previously observed that RXRA DT448/9PP was associated with increased binding to an NCOR2 co-repressor (nuclear receptor corepressor 2, formerly SMRT) peptide in a GST pull-down assay. ${ }^{16} \mathrm{We}$ included this variant as part of the screen to evaluate corepressor requirements. DT448/9 is positioned at the end of the turn between helix 11 and 12. Helix 11 directly binds to co-repressors and co-activators. ${ }^{17}$ Helix 12 (the AF2 domain) adopts an extended and unstructured conformation in the absence of ligand and assumes a repositioned and helical conformation following ligand binding (Figure 1A). ${ }^{18}$ Unexpectedly, retroviral expression of RXRA DT448/9PP (MSCV-RXRA DT448/9PP-IRES-mCherry) in RXR-KO KMT2A-MLLT3 leukemia cells induced the normally round cells to form adherent clumps that were $\mathrm{mCherry}^{+}$on the tissue culture plate (Figure $1 \mathrm{~B})$. mCherry ${ }^{+}$cells were also associated with loss of leukemic colony-forming capacity, lack of proliferation, and macrophage-like cytomorphology with ruffled borders and vacuoles (Figure 1C-E). These effects were absent in RXR-KO KMT2A-MLLT3 cells retrovirally transduced with RXRA WT (MSCV-RXRA-IRES$m$ Cherry), with other tested RXRA domain deletions or point mutations, or with treatment combinations of RXRA and RARA activating ligands ${ }^{5}$ (Figure $1 \mathrm{~B}$ ). In addition, we found that retroviral expression of RXRA DT448/9PP (MSCV-RXRA DT448/9PP-IRES-mCherry) in KMT2AMLLT3 WT leukemia cells induced the same phenotype as that observed in RXR-KO KMT2A-MLLT3 leukemia cells (lack of proliferation and macrophage-like cytomorphology) (data not shown). We examined the resulting proteins on western blot after transduction into KMT2A-MLLT3 WT cells (Figures 1F). RXRA DT448/99PP was associated with a similar sized band as wild-type RXRA, but it appeared resistant to the proteolytic cleavage that results in the formation of a truncated $36 \mathrm{kDa}$ fragment. ${ }^{19}$ Endogenous Rxra levels were significantly lower than levels of retrovirally expressed RXRA (Figure 1G). Thus, retroviral transduction led to similar levels of expression of RXRA WT and RXRA DT448/9PP, at supraphysiological protein levels compared to endogenous RXRA. 
A

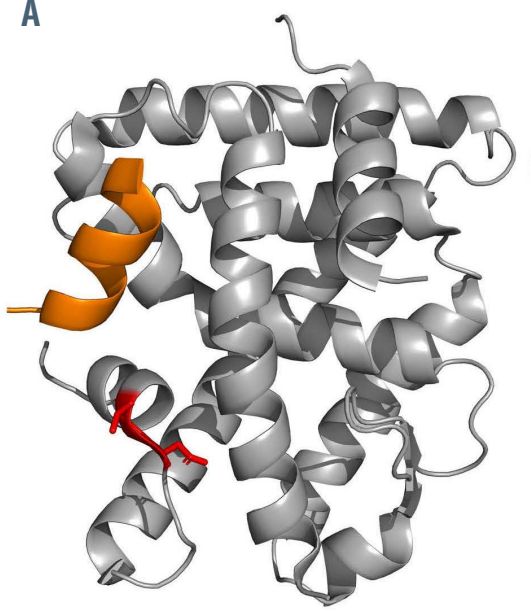

B

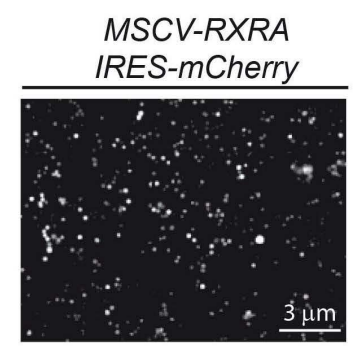

mCherry
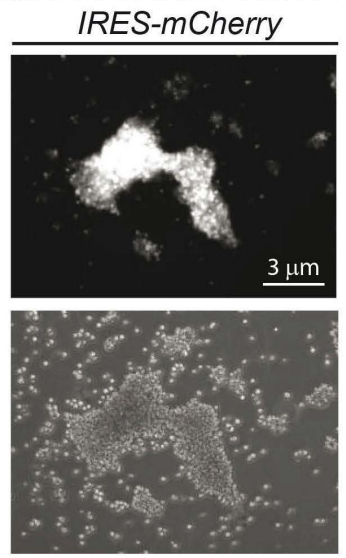

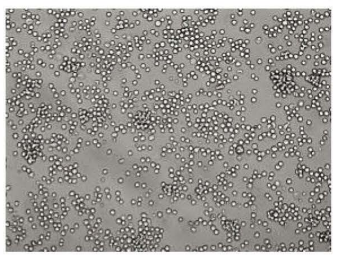

Brightfield
C

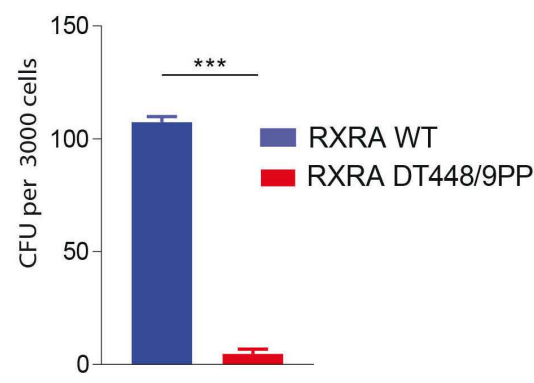

E

RXRA WT (Endogenous)

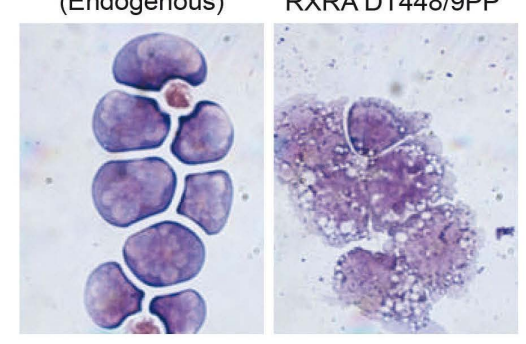

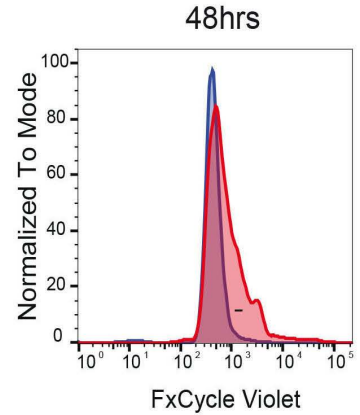

F RXRA RXRA WT
RXRA DT
$72 \mathrm{hrs}$

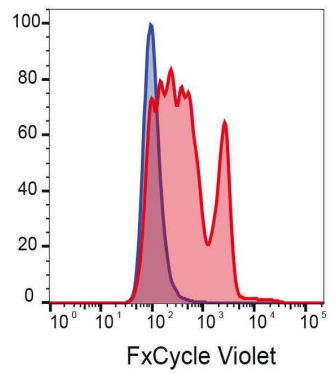

G
- RXRA DT448/9PP (mCherry + cells) a RXR-KO (mCherry - cells)

$\mathrm{H}$

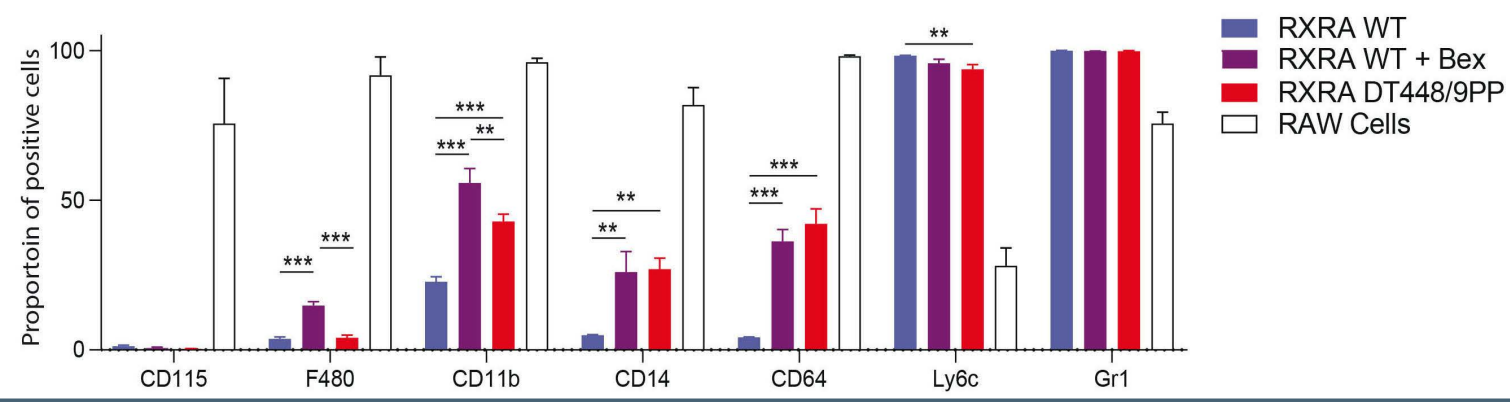

Figure 1. Monocytic maturation induced by RXRA DT448/9PP. (A) DT448/9 amino acids are highlighted in red within the structure of the RXRA ligand binding domain (PDB 4K4J). An Ncoa2 peptide is highlighted in orange. Note that DT448/9 occurs at the beginning of helix 12, the AF2 domain, and the rigid proline substitution could enhance helix formation. (B, C) RXR-KO KMT2A-MLLT3 leukemia cells were transduced with MSCV-RXRA DT448/9PP-IRES-mCherry or with MSCV-RXRA WTIRES-mCherry. Cells were evaluated under fluorescent and light microscopy at $72 \mathrm{~h}$. (B) Transduced cells were sorted for mCherry ${ }^{+}$cells and plated in methylcellulose, and colonies assessed in technical triplicates on day 7. Statistical significance evaluated using the $t$-test, $* * * P<0.001$ (C). (D) RXR-KO KMT2A-MLLT3 leukemia cells were transduced with MSCV-RXRA DT448/9PP-IRES-mCherry, stained with FxCycle violet, and retention of the dye was assessed by flow cytometry at the indicated time points comparing mCherry (RXRA DT 448/9 PP) versus mCherry- (RXR-KO) cells. (E) Cytomorphology of mCherry ${ }^{+}$(RXRA DT 448/9 PP) versus mCherry- (RXRA WT endogenous) sorted KMT2A-MLLT3 leukemia cells after transduction with MSCV-RXRA DT448/9PP-IRES-mCherry. (F) RXR-KO KMT2A-MLLT3 leukemia cells were transduced with MSCV-RXRA DT448/9PP-IRES-mCherry or with MSCV-RXRA-IRES-mCherry and protein expression was evaluated through western blot analysis using anti-RXRA antibody (H-10, Santa Cruz). GAPDH was used as a loading control. (G) KMT2A-MLLT3 WT leukemia cells were transduced with MSCV-RXRA WT-IRES$m$ Cherry as indicated, and protein expression was evaluated through western blot analysis using anti-RXRA antibody (5388, Cell Signaling). GAPDH was used as a loading control. (H) KMT2A-MLLT3 WT leukemia cells were transduced as indicated and analyzed by flow cytometry. RXRA WT cells were analyzed with and without treatment with $250 \mathrm{nM}$ bexarotene for $24 \mathrm{~h}$. RAW 264.7 cells were used as a positive staining control, but not included in the statistical comparisons. Statistical significance was evaluated using analysis of variance with the Tukey correction for multiple comparisons, $* P<0.05$. $* * P<0.01$. $* * * P<0.001$. 
Because the RXRA DT448/9PP-transduced cells acquired cell adhesion properties and ruffled borders suggestive of macrophage-like maturation, we assessed immunophenotypic changes. Bexarotene treatment of RXRA WT transduced cells resulted in an increased percentage of cells expressing F4/80, CD11b, CD14, and CD64, as determined by flow cytometry, and increased the median fluorescence intensity (MFI) of F4/80, CD11b, CD14, CD64, Ly6c, and Gr1, but had little effect on CD115 expression. RXRA DT448/9PP transduction resulted in increased percentages of cells expressing CD11b, CD14, and CD64, with increased MFI of CD11b, CD14, CD64, Ly6c and Gr1, consistent with myeloid and monocytic maturation, but lacking the classical macrophage marker CD115 (Figure 1H, Online Supplementary Figure S1).

Transduction of RXRA DT448/9PP, but not RXRA WT into two KMT2A-MLLT3-associated human cells (THP1 and MonoMac-6) also resulted in adherence to plastic with the development of podocytes and ruffled borders (Online Supplementary Figure S2). These effects required 4 to 6 days to emerge, whereas adhesion and cell clumping in murine KMT2A-MLLT3 cells typically required only 3 or 4 days. In contrast, $\mathrm{Kit}^{+}$murine bone marrow cells cultured in stem cell media (stem cell factor, interleukin 3, thrombopoietin, Flt3L) and K562 cells were resistant to the effects of RXRA DT448/9PP, and the cells remained round and non-adherent (Online Supplementary Figures S2D and S3A, B).

\section{RXRA DT448/9PP is constitutively active}

We assessed the functional activity of RXRA DT448/9PP using multiple reporter systems. First, we used a UAS/Gal4 reporter. ${ }^{11}$ KMT2A-MLLT3 WT leukemia cells derived from UAS-GFP bone marrow cells were retrovirally transduced with a fusion of the Gal4 DNA binding domain and the RXRA wild-type ligand-binding domain (Gal4-RXRA) or a fusion of the Gal4 DNA-binding domain and the RXRA DT448/9PP ligand-binding domain (Gal4-RXRA DT448/9PP). Gal4-RXRA DT448/9PP resulted in constitutive activity uninfluenced by increasing concentrations of bexarotene (Figure 2A). Constitutive activity of RXRA DT449/8PP was also observed in $\mathrm{Kit}^{+}$UAS-GFP bone marrow cells transduced with Gal4-RXRA retroviruses (Online Supplementary Figure S3C).

Second, we evaluated three different luciferase reporters in 293T cells. 293T cells are known to express endogenous RXRA as well as various of its partners; RARA, PPARG, and VDR ${ }^{20}$ Reporters assays included a synthetic direct repeat 1 (DR1) peroxisome proliferator response element (PPRE); a DR1 response element from the $A p \circ A 1$ promoter; and a DR5 response element from the $R A R B$ promoter. We noted that $R X R A$ DT448/9PP transfection consistently led to ligand-independent activation of all three constructs (Figure 2B). A control RXRA construct contained a deletion of the AF2 domain (RXRA $\triangle \mathrm{AF} 2$ ), and this construct is unresponsive to ligand. Using this approach we assessed whether RXRA DT448/9PP might be sensitive to inhibition by two pan-RXR antagonists, HX531 and UVI3003. Neither compound inhibited activation of the UAS/Gal4 reporter or the RARE-Luc reporter (Figure 2C).

We used a mammalian two-hybrid assay to assess the interaction of RXRA DT448/9PP with the co-activator PGC1 $\alpha$, noting constitutive, ligand-independent binding (Figure 2D, E). This activity remained present with the L2/3A variant (L147A, L148A, L210A, L211A), ${ }^{21}$ which contains point mutations in the critical LXXLL motifs required for ligand-dependent interactions of PGC1 $\alpha$ with nuclear receptors. Thus, RXRA DT448/9PP appears to constitutively engage PGC1 $\alpha$ using domains outside the canonical $\mathrm{N}$ terminal LXXLL motifs.

\section{Comparison with other active RXRA variants}

Two other $R X R A$ variants with constitutively active properties have been described. First, mouse Rxra F318A exhibits constitutively active phenotypes. ${ }^{22}$ However, when a crystal structure was generated, the ligand-binding pocket contained oleic acid, and Rxra F318A activity could be inhibited by the pan-RXR antagonist HX531, suggesting that the mutation leads to augmented responsiveness to a natural ligand present in the tissue culture, and is not completely ligand-independent. ${ }^{23}$

We assessed the sensitivity of RXRA DT448/9PP to two pan-RXR antagonists (HX531 and UVI3003) across a series of assays. In RXR-WT KMT2A-MLLT3 leukemia cells transduced with $R X R A$ DT448/9PP, cell adhesion, clumping, and loss of proliferation phenotypes were not abrogated by either compound (Online Supplementary Figure S4).

To further assess whether RXRA DT448/9PP phenotypes may result from hyper-responsiveness to intracellular natural ligands, we mutated two amino acids (R316 and L326) that form critical ionic bonds with the carboxylic acid group in ligands (e.g., bexarotene, 9-cis retinoic acid, and long-chain fatty acids). ${ }^{5}$ RXRA R316A/L326A has been previously shown to abrogate ligand-dependent activation and is unable to rescue response to bexarotene in RXR-KO KMT2A-MLLT3 leukemia cells. ${ }^{5,24}$ Retroviral expression of the compound variant RXRA R316A/L326A/DT448/9PP again led to overexpression on western blot and resistance to proteolytic cleavage, but did not abrogate cell clumping, loss of proliferation, or loss of colony formation (Figure 3A-D).

Second, recurrent $R X R A$ hot-spot mutations (S427F/Y) occur in patients with bladder cancer, and these augment the activity of the PPARG:RXRA heterodimer, but are not capable of independently activating RXRA reporters. ${ }^{25}$ Retroviral expression of RXRA S427F also led to strong overexpression of the variant, which retained sensitivity to proteolytic cleavage, although cell adhesion, cell clumping, cell proliferation, and colony-forming properties were not altered (Figure 3E-H).

To determine whether similar heterodimerization with PPARG or with other nuclear receptors may play essential roles in the activity of RXRA DT448/9PP, we evaluated phenotypes using a series of receptor antagonists. Concurrent treatment with potent antagonists of retinoic acid receptors, LXR, PPARA, and PPARG did not affect clumping, adhesion to plastic, or proliferation phenotypes (Online Supplementary Figure S5), demonstrating that the DT448/9PP creates a nonpermissive receptor that is functionally active, independently of other activated nuclear receptors.

\section{RXRA DT448/9PP inhibits leukemic engraftment and expansion}

KMT2A-MLLT3 WT cells were transduced with RXRA WT or RXRA DT448/9PP retroviruses labeled with IRES$m$ Cherry cassettes (Figure 4A). To limit maturation effects in vitro, the populations were immediately transplanted into recipient mice and residual cells were subsequently analyzed by flow cytometry. After 4 weeks of engraftment and expansion, leukemia cells in the peripheral blood were assessed and we observed that mice transplanted with 
RXRA WT displayed increased leukocytosis, an increase in neutrophils, and a decrease in lymphocytes compared to mice transplanted with $R X R A$ DT448/9PP-transduced cells (Figure 4B-D). Compared with the pre-engraftment population (RXRA WT, 55\% mCherry'; RXRA DT448/9PP, 62\% mCherry $)^{+}$, at 4 weeks there was reduced engraftment in RXRA DT448/9PP cells relative to RXRA WT cells: RXRA WT average of $7.6 \%$ mCherry $^{+}$cells (range, $0.94 \%-16.4 \%$ ) versus RXRA DT448/9PP $0.25 \%$ mCherry $^{+}$cells (range,
0.095\%-0.36\%) (Figure 4E). When we assessed moribund mice transplanted with cells transduced with RXRA WT, we observed further reduction in the absolute proportion of $\mathrm{mCherry}^{+}$cells in the bone marrow rather than an expansion: RXRA WT, average 1\% (range, 1.21\%-1.46\%); RXRA DT448/9PP, average 0.741 (range, $0.022 \%-1.46 \%$ ) (Figure 4G). Survival was shorter in mice transplanted with $R X R A$ WT-transduced cells than in those transplanted with $R X R A$ DT448/9PP-transduced cells (Figure 4H). At the time of sac-
A

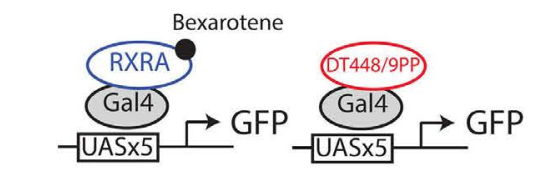

B
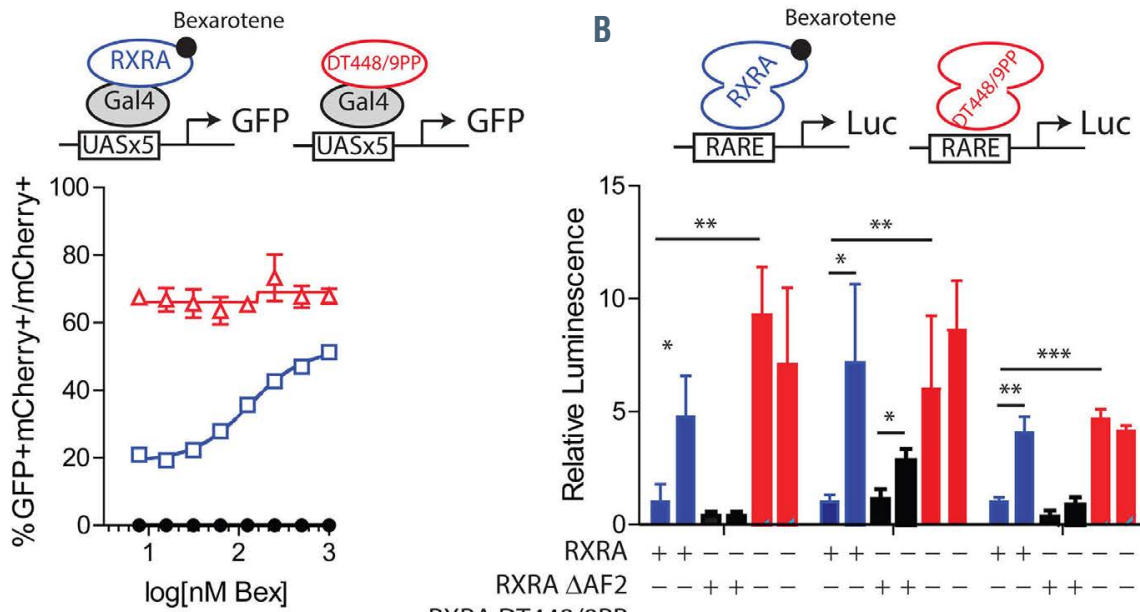

$\triangle$ Gal4-RXRA DT448/9PP

$-\square-$ Gal4-RXRA WT

$\rightarrow$ Mock

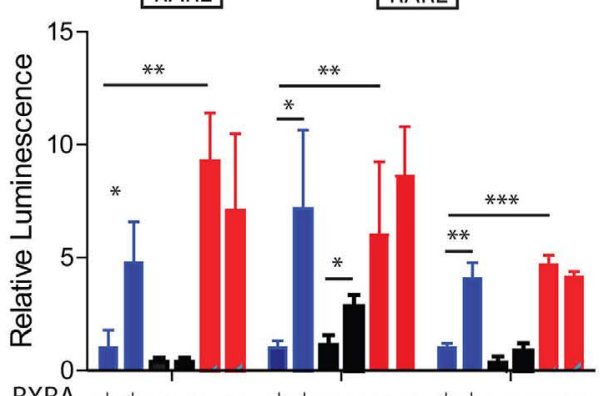

RXRA ++--- ++----++----

RXRA $\triangle \mathrm{AF} 2--++----++----++--$

RXRA DT448/9PP ----++----++----++

Bexarotene $\frac{-+-+-+}{\text { PPRE-LuC }} \frac{-+-+-+}{\text { ApoA1-LuC }} \frac{-+-+-+}{\text { RARE-LuC }}$
C

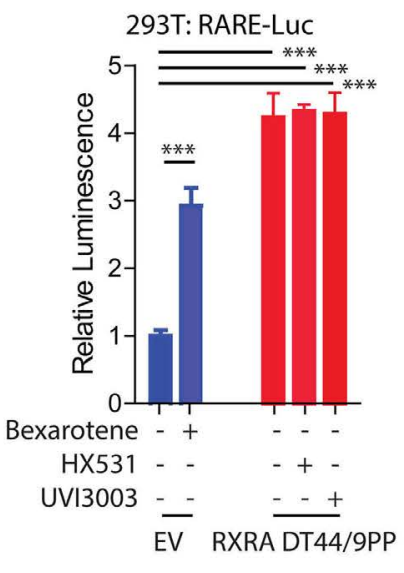

D
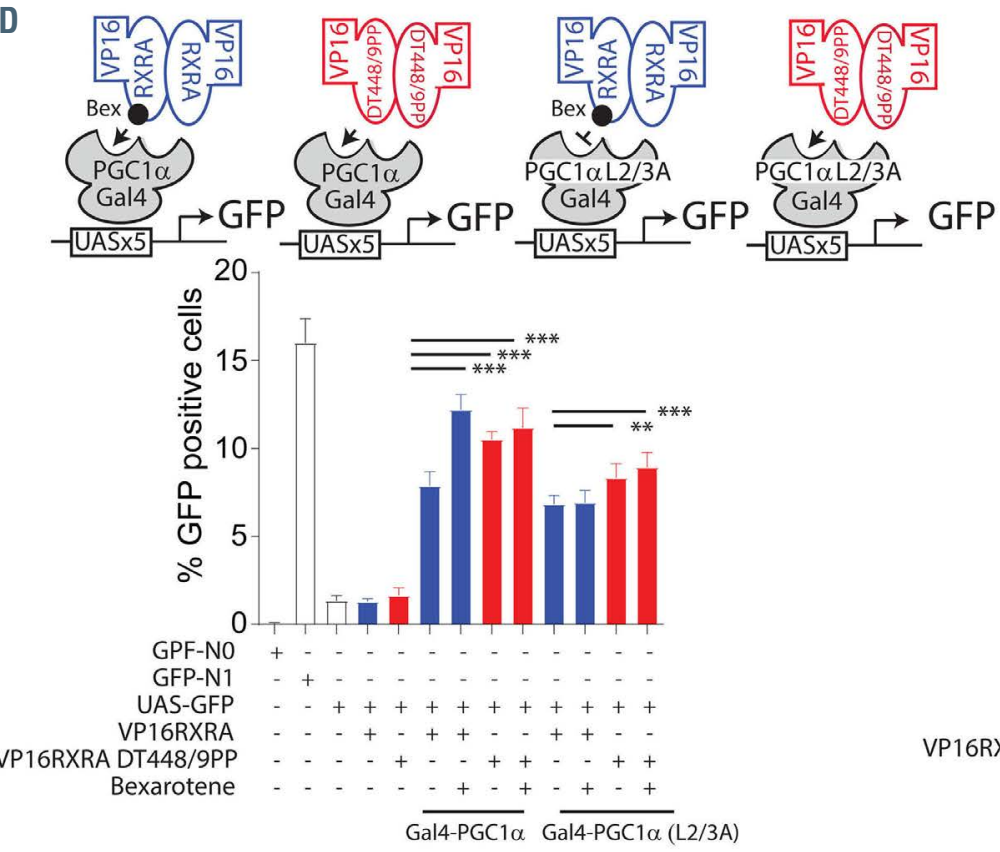

E

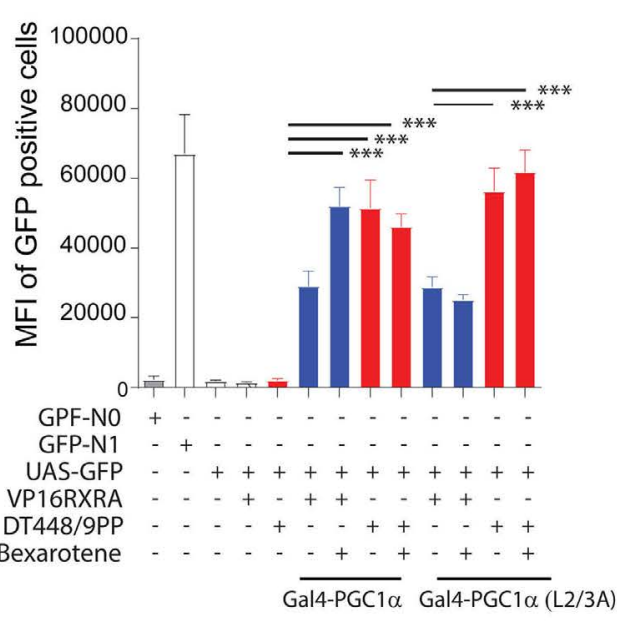

Figure 2. Constitutive activity of RXRA DT448/9PP. (A) KMT2A-MLLT3 WT leukemia cells derived from UAS-GFP bone marrow cells were transduced with MSCV-Gal4RXRA DT448/9PP-IRES-mCherry or MSCV-Gal4-RXRA-IRES-mCherry, treated with increasing concentrations of bexarotene, and the ratio of GFP' cells (responding) to total mCherry cells (capable of responding) was determined. Data from biological duplicates are shown. (B, C) 293T cells were transfected with the indicated plasmids, treated with bexarotene, HX531, or UVI3003 (all $1 \mu \mathrm{M}$ ), and luciferase was measured after $40 \mathrm{~h}$. Data from biological triplicates are shown. PPRE: peroxisome proliferator response element. ApoA1: DR1 element from the ApoA1 promoter. RARE: retinoic acid receptor response element from the RARB promoter. The $\triangle \mathrm{AF} 2$ deletion acts as a negative control. (D, E) Mammalian two-hybrid. 293T cells were transfected with the indicated plasmids, treated with bexarotene and GFP was measured by flow cytometry after $40 \mathrm{~h}$. Data from biological triplicates are shown. GFP-N1: a positive control CMV-GFP expression vector. GFP-NO: a negative control derived from GFP-N1 after the deletion of the CMV promoter. Statistical significance was evaluated using analysis of variance with the Bonferroni comparison to control, $* P<0.05, * * P<0.01, * * * P<0.001$. 
rifice, all but one mouse had developed splenomegaly (average spleen weight $0.5 \mathrm{~g}$ ) and bone marrow lacked erythroid elements, features that are typical of overwhelming secondary leukemic engraftment (Online Supplementary Figure S6).

\section{Transcriptional consequences of RXRA DT448/9PP}

To assess the maturation effects of RXRA DT448/9PP more comprehensively and to investigate the overlapping consequences with RXRA WT activation by bexarotene, KMT2A-MLLT3 WT leukemia cells were transduced with RXRA WT or RXRA DT448/9PP and the transcriptional profiles of the transduced cells were assessed by RNA sequencing (Figure $5 \mathrm{~A}$ ). We noted significant overlap of regulated genes between RXRA WT cells treated with bexarotene and cells transduced with RXRA DT448/9PP (Figure 5B-D). Of 598 genes that were upregulated in RXRA WT cells treated
A

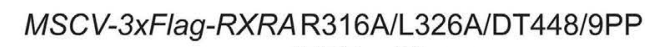
IRES-mCherry
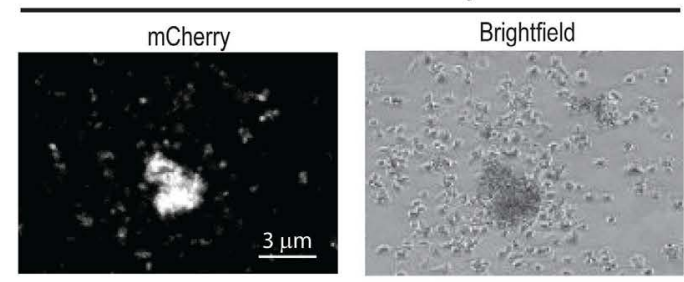

C

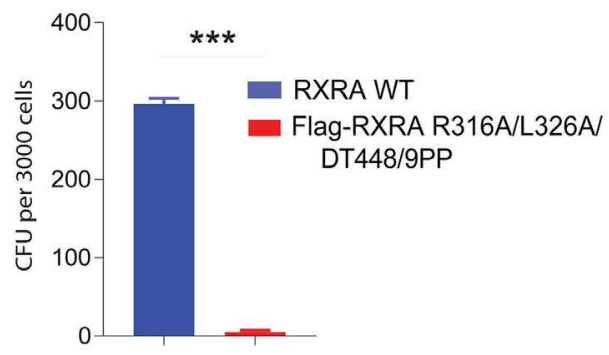

E
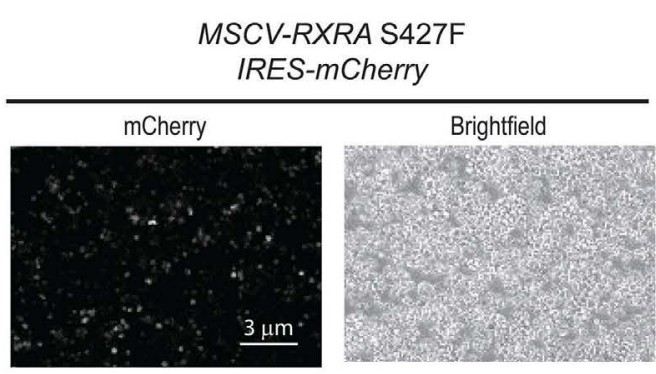

G

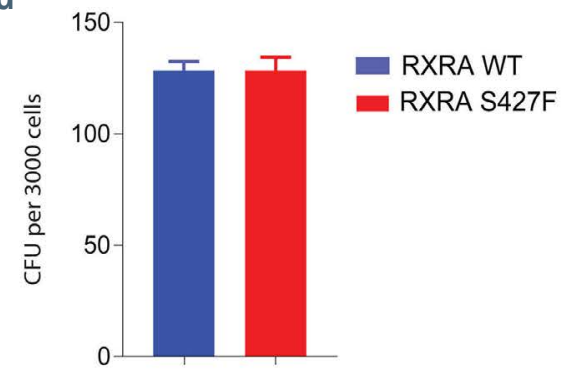

D

$\mathbf{F}$

H
B

B $\quad 72 \mathrm{~h}$

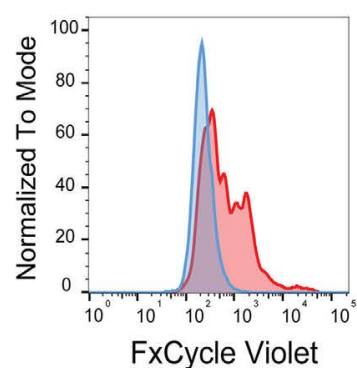

RXRA R316A/L326A/DT448/9PP

(mCherry + cells)

- RXR-KO

(mCherry - cells) $\begin{array}{llll}\text { RXRA WT } & + & - & - \\ \text { RXRA DT448/9PP } & - & + & - \\ \text { 3XFlag-RXRA R316A/L326A/ } & - & - & + \\ \text { DT448/9PP } & \end{array}$
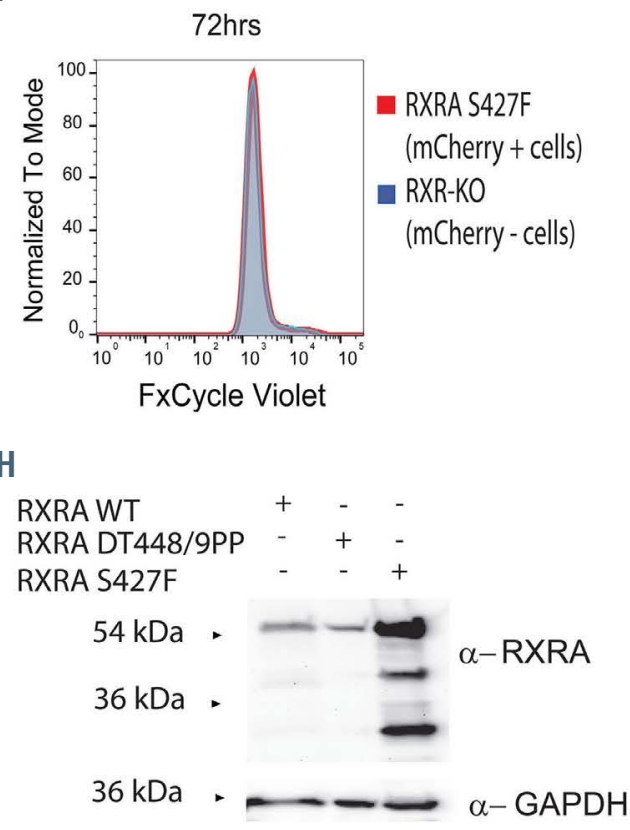

Figure 3. Comparison with other RXRA variants with activity. KMT2A-MLLT3 WT leukemia cells were transduced with MSCV-3xFlag-RXRA R316A/L326A/DT448/9PPIRES-mCherry or with MSCV-RXRA S427F-IRES-mCherry and evaluated under fluorescent and light microscopy at $72 \mathrm{~h}$ (A and E). RXR-KO KMT2A-MLLT3 leukemia cells were transduced with MSCV-3xFlag-RXRA R316A/L326A/DT448/9PP-IRES-mCherry or MSCV-RXRA S427F-IRES-mCherry, stained with FxCycle Violet, and retention of the dye was assessed by flow cytometry at the indicated time points (B and F). RXR-KO KMT2A-MLLT3 leukemia cells were transduced with MSCV-RXRA WT-IRES-mCherry or MSCV-3xFlag-RXRA R316A/L326A/DT448/9PP-IRES-mCherry or MSCV-RXRA S427F-IRES-mCherry, mCherry+ cells were then sorted and plated in methylcellulose, and colonies assessed in technical triplicates on day 7 (C and G). RXR-KO KMT2A-MLLT3 leukemia cells were transduced with MSCV-RXRA-IRESmCherry or MSCV-RXRA DT448/9PP-IRES-mCherry or MSCV-RXRA S427F-IRES-mCherry and protein expression was evaluated through western blot analysis using anti-RXRA antibody (H-10, Santa Cruz). GAPDH was used as a loading control (D and H). Statistical significance was evaluated using the $t$-test, $* * \star P<0.001$. 
with bexarotene, 525 overlapped with those of RXRA DT448/9PP-transduced cells. Likewise, of 74 downregulated genes in RXRA WT cells treated with bexarotene, 28 overlapped with those in RXRA DT448/9PP-transduced cells.

Gene ontology pathway analysis showed enrichment of lipid metabolism and immune system signaling and downregulation of RNA processing (Online Supplementary Tables S1 and S2). Gene set enrichment analysis of a curated list of genes regulated during myeloid development ${ }^{26}$ indicated that treatment of RXRA WT cells with bexarotene and expression of RXRA DT448/9PP both lead to a myeloid differentiation phenotype (upregulation of "Up" genes and downregulation of "Down" genes) compared to untreated WT cells (Figure 5E, F).

Within this set of differentially expressed genes, we evaluated transcripts associated with cell surface proteins. Sixty-seven cell surface transcripts were differentially expressed, according to RNA sequencing, between RXRA WT cells versus RXRA WT cells treated with bexarotene or RXRA DT448/9PP-transduced cells (Online Supplementary Figure $S 7 A$ ). As in the overall RNA sequencing analysis, these genes were associated with similar or greater upregulation in DT448/9PP-transduced cells than in the wild-type cells treated with bexarotene (Online Supplementary Figure

A

MSCV-RXRA WT-IRES-mCherry

or

MSCV-RXRA DT448/9PPIRES-mCherry

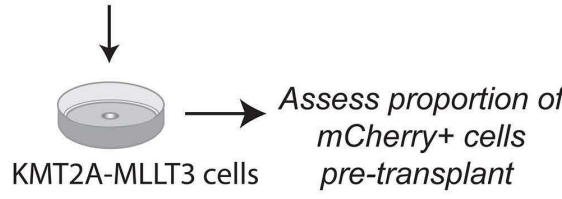

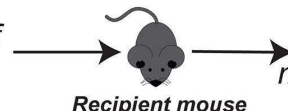

Recipient mouse
Assess proportion of

Cherry+ cells at week 4 post-transplant
B

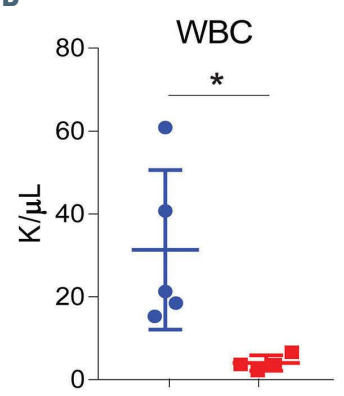

E

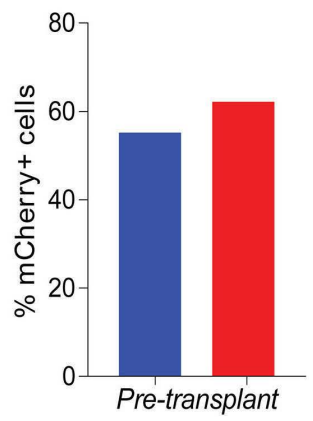

C

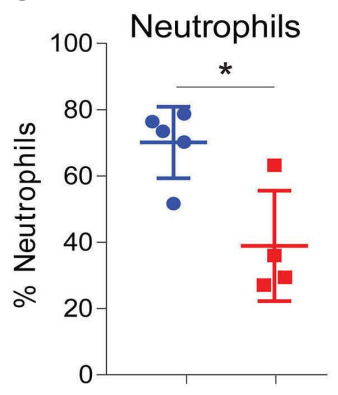

F

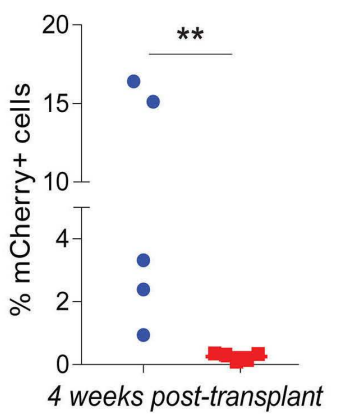

D

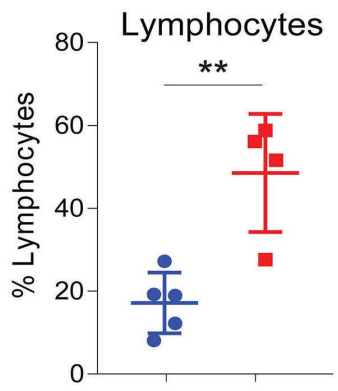

G

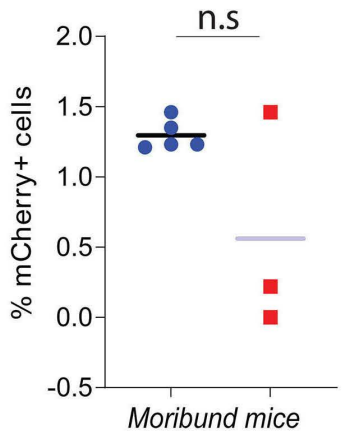

H

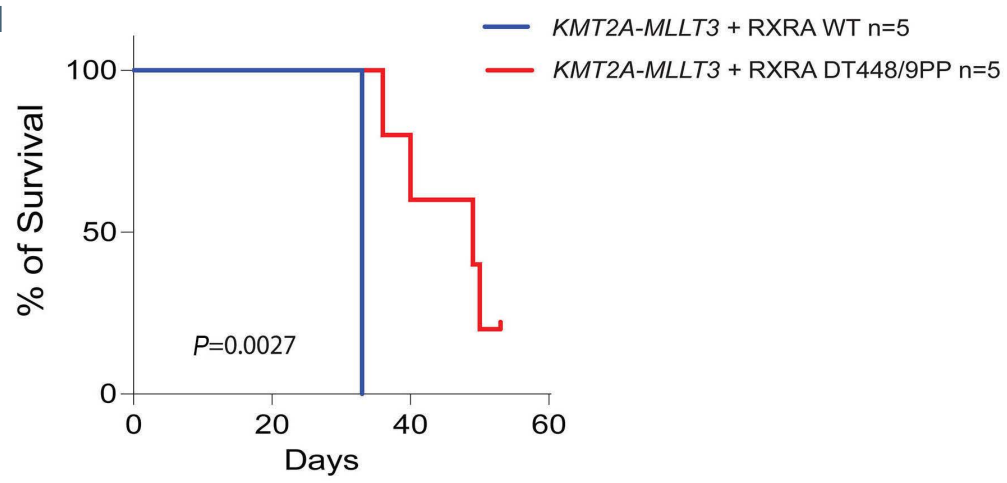

- RXRAWT

- RXRA DT448/9PP

\section{Survival}


$S 7 B-F)$. The list of upregulated genes included CD14 and CD64 (Fcgr1), which at the protein level were observed to increase by immunofluorescence (Figure $1 \mathrm{H}$, Online Supplementary Figure S1C-D). The most highly differentiated transcript out of the cell surface markers was the complement factor C3 (Online Supplementary Figure S7D), and additional complement-receptors (C5ar1 and CD59a) also underwent upregulation with RXRA activation (Online Supplementary Figure $S 7 B, F$ ), which correlate with monocytic differentiation. We also noted a variety of upregulated integrins (Bcam, Ceacam1, Itgam, Itgb2, Itgb2l, and Itgb7), and these may play a role in the aggregation phenotype of the DT448/9PP variant (Online Supplementary Figure S7C-F).

While the majority of transcripts that were up- or downregulated overlapped between RXRA WT cells treated with bexarotene and RXRA DT448/9PP-transduced cells (Figure 5B-D), there were 11 genes that were differentially expressed between these two groups (Online Supplementary Figure S8A). In five, RXRA DT448/9PP augmented the bexarotene-induced response: (upregulated Vsig8, Fcgr1 (Cd64), Camp, Gp6; downregulated: Pik3ip1) (Online Supplementary Figure S8B). The other six displayed three different patterns: not expressed in treated or untreated RXRA WT cells and increased in DT448/9PP-transduced cells (Gpr84, S100a8); downregulated from untreated to treated RXRA WT cells and expressed in DT448/9PP-transduced cells (Mpo, Gm28438, Gm10359); or not expressed in untreated RXRA WT with an increase upon bexarotene treatment but not with DT448/9PP (Cited1) (Online Supplementary Figure S8C). This list is too small for pathway
A

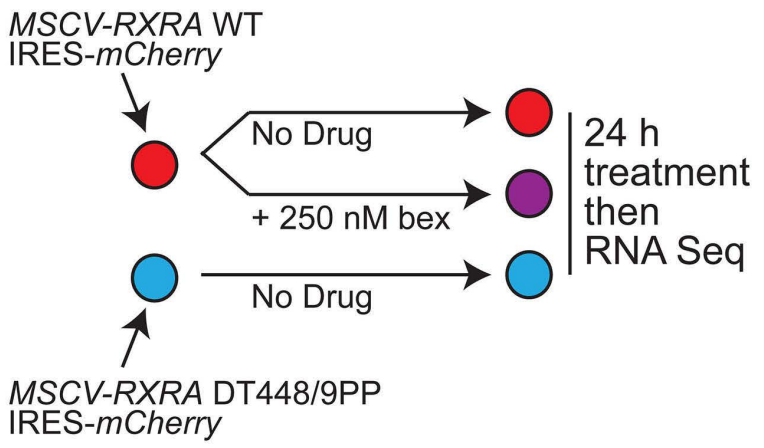

C

73

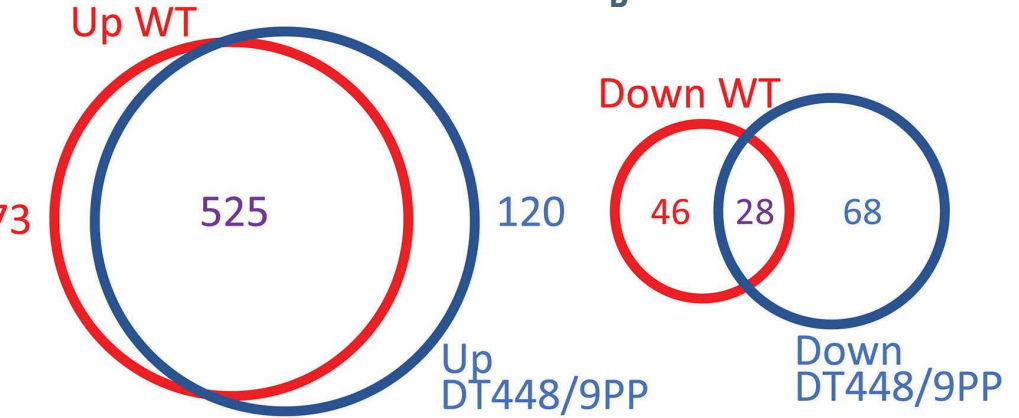

B

Differentially expressed genes

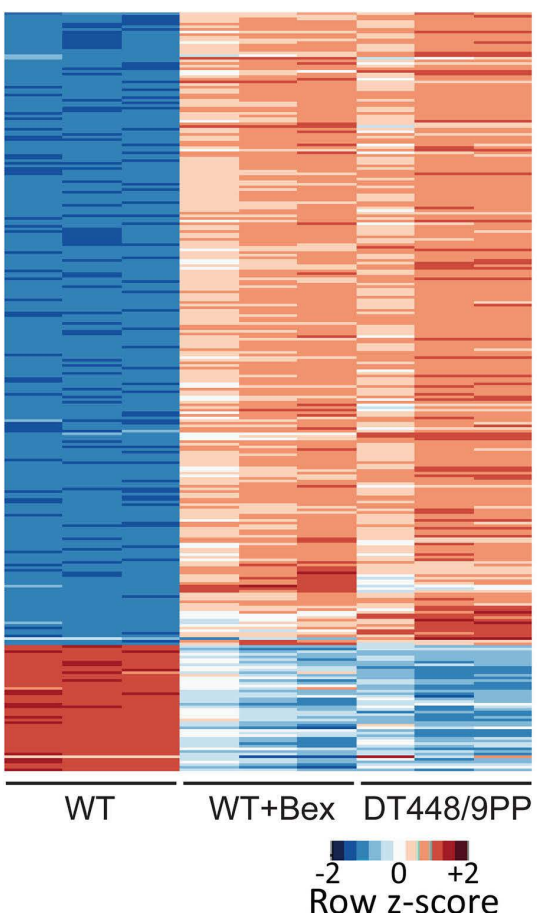

E

Myeloid Cell Development Up

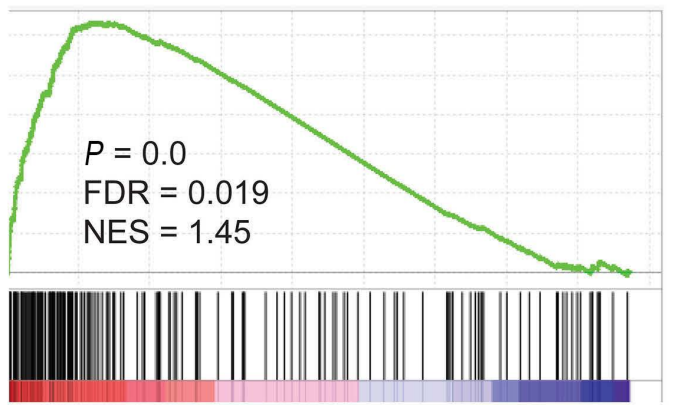

$\mathbf{F}$

Myeloid Cell Development Down

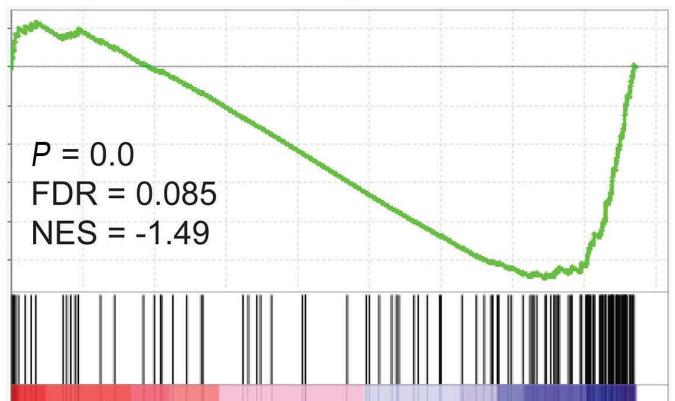

Figure 5. RXRA DT448/9PP transcriptome changes. (A) Schema of experimental design: KMT2A-MLLT3 WT leukemia cells were transduced with the indicated viruses and cultured for $24 \mathrm{~h}$ with or without $250 \mathrm{nM}$ bexarotene; mCherry cells were sorted, and total RNA was isolated for RNA sequencing analysis. (B) Heatmap of differentially expressed genes (DEG) between all three groups. Each condition was evaluated by biological triplicates. WT: RXRA WT cells; WT + bex: RXRA WT cells treated with bexarotene; DT448/9PP: RXRA DT448/9PP-transduced cells. (C) Venn diagram with the number of upregulated unique DEG from RXRA WT cells versus RXRA WT cells treated with bexarotene (red) and RXRA WT cells versus RXRA DT448/9PP-transduced cells (blue) and DEG common to both groups (purple). (D) Venn diagram with the number of downregulated unique DEG from RXRA WT cells versus. RXRA WT cells treated with bexarotene (red) and RXRA WT cells versus RXRA DT448/9PP-transduced cells (blue) and DEG in common between both groups (purple). (E, F) Gene set enrichment analysis comparing the untreated RXRA WT cells to the bexarotene-treated and DT448/9PP-transduced cells upregulated (E) and downregulated (F) during myelopoiesis (published gene sets from Brown, et al. ${ }^{26}$ ). Heatmaps are shown as row z-scores with the color key below the panel (B). FDR: false discovery rate; NES: normalized enrichment score. 
analysis, but manual curation found that the most of these 11 transcripts are related to immune function and activity.

\section{Discussion}

Retinoic $\mathrm{X}$ receptors and retinoic acid receptors have been shown to influence myeloid maturation. . $2,4,27,28_{\text {RXRA }}$ protein levels and activity have been linked to cell fate decisions at the neutrophil versus monocyte decision, ${ }^{29}$ and deletion of Rxra and Rxrb prevents osteoclast maturation. ${ }^{12}$ and augments KMT2A-MLLT3 leukemogenesis and cell expansion in vivo, ${ }^{5}$ suggesting a role for retinoic $X$ receptors in regulating myeloid maturation.

RXRA DT448/9PP is a serendipitously discovered mutation that results in constitutive activity, leukemic cell maturation, and loss of proliferative capacity. This variant demonstrates ligand-independent activation, augmented co-activator binding, induced maturation transcripts by RNA sequencing, and reduced engraftment in vivo. To our knowledge, RXRA DT448/9PP has not been spontaneously observed in cancer or other pathological states.

Two other RXRA mutations with activating phenotypes have been described. Mouse Rxra F318A has increased transcriptional activity, potentially via increased responsiveness to cell-available oleic acid. ${ }^{22}$ However, Rxra F318A retains sensitivity to RXRA antagonists, whereas RXRA DT448/9PP did not (Figure 2C, Online Supplementary Figure S2). Furthermore, R316 and L326 are critical amino acids that interact directly with bound ligand and are required for ligand activation of RXRA. ${ }^{5,24}$ Combining these ligandblocking mutations with DT448/9PP, we noted retained KMT2A-MLLT3 maturation phenotypes (Figure 3A-C), further suggesting ligand-independent activity of RXRA DT448/9PP. Recurrent RXRA hot-spot mutations (S427F/Y) have been noted in patients with bladder cancer. These mutations augment the activity of the PPARG:RXRA heterodimer and are not capable of independently activating RXRA in reporter assays. ${ }^{25}$ We previously found that combinations of RARA and RXRA ligands lead to leukemic maturation and apoptosis, whereas PPARG and RXRA ligands did not. ${ }^{5}$ Here we found that RXRA S427F did not recapitulate leukemia maturation phenotypes in KMT2AMLLT3 leukemia cells (Figure 3E-G), and the PPARG antagonist T0070907 did not abrogate RXRA DT448/9PP phenotypes (Online Supplementary Figure S5). Thus, in contrast to Rxra F318A and RXRXA S427F, RXRA DT448/9PP may be constitutively active, independently of endogenous, available, natural ligands, and also may not depend on activation through PPARG:RXR heterodimers or other major nuclear receptor heterodimers.

RXRA DT448/9PP constitutive activity may be related to at least two phenotypes. RXRA DT448/9PP is resistant to enzymatic cleavage, and this may enable augmented functional protein levels (Figures $1 \mathrm{~F}$ and $3 \mathrm{D}, \mathrm{H}$ ), but cannot explain resistance to pan-RXR antagonists or co-mutation with R316A/L326A (Figures 2C and 3A-C, Online Supplementary Figure S4). RXRA DT448/9PP was also associated with ligand-independent co-activator binding (Figure 2D), which may enable augmented, or even constitutive activity. A limitation of these studies is that they require a retroviral overexpression system and therefore the effect of DT448/9PP RXRA at physiological levels is unknown.

Not all cells tested were susceptible to RXRA DT448/9PP. The two human myelomonocytic acute myeloid leukemia lines with KMT2A-MLLT3 (THP1 and MonoMac-6) were susceptible, whereas the heterogeneous stem/progenitor populations of $\mathrm{Kit}^{+}$murine bone marrow cells and the blast phase chronic myeloid leukemia cell line K562 were not (Online Supplementary Figures S2 and S3). Maintained in stem cell cytokines, $\mathrm{Kit}^{+}$bone marrow cells have multipotent potential and their medium lacks cytokines that might provide monocytic maturation support. K562 cells are susceptible to erythroid maturation stimuli rather than myeloid maturation. Other groups have observed differences in sensitivity and resistance to retinoids across cell lines. ${ }^{9,30,31}$ Thus, different external signals and/or internal priming states may affect the susceptibility to retinoids and to RXRA DT448/9PP.

We have previously noted that Rxra and Rxrb expression negatively regulates KMT2A-MLLT3 leukemia and that these cells are exposed to low levels of natural RXRA ligands in vivo.,32 Similarly, here we found that cells that overexpress RXRA WT consistently engraft in recipient mice, but are associated with a competitive disadvantage relative to untransduced, mCherry-negative cells (Figure 4). This phenotype was augmented by transduction with RXRA DT448/9PP, which further limited engraftment and leukemic outgrowth in vivo, again suggesting the potential of activated retinoic $\mathrm{X}$ receptors to inhibit growth of leukemia cells in vivo.

RXRA DT448/9PP resulted in several maturation-related phenotypes, including lack of proliferation, loss of colony formation, acquisition of ruffled borders and podocytes, and increased expression of cell surface markers of myeloid and monocytic maturation. Transcriptional analysis of bexarotene-treated cells versus RXRA DT448/9PP-transduced cells suggested strong overlapping myeloid and monocytic maturation signatures, consistent with ligandindependent, constitutively active effects of DT448/9PP. Many maturation-related transcripts were more effectively induced by RXRA DT448/9PP than by bexarotene, and a few myeloid-related transcripts were uniquely induced by RXRA DT448/9PP. Thus, DT448/9PP may more effectively activate the same loci as ligand-activated RXRA, and activity at select novel loci may enable additional phenotypes.

Like other nuclear receptors, the retinoic X receptors are ligand-dependent transcription factors and their function and activity change from transcriptional repressors to transcriptional activators in the presence of active ligands. Multiple natural ligands have been proposed for the retinoic $\mathrm{X}$ receptors, ${ }^{6}$ and it is often difficult to know which cells and settings contain active, natural ligands, and when an observed effect of retinoic X receptors may be related to the receptor function in the absence versus presence of ligand. A constitutively active variant provides a helpful genetic comparator and could be used with diverse forms of previously characterized non-functional (e.g., $\triangle \mathrm{DBD}, \mathrm{E} 153 \mathrm{G} / \mathrm{G} 154 \mathrm{~S}$ ) and ligand-non-responsive variants (e.g., $\triangle \mathrm{AF} 2$, R316A/326A).

Retinoid receptors have been an attractive therapeutic target in acute myeloid leukemia; however their effects in clinical trials have been modest. The maturation effects (loss of proliferation and colony-forming potential, and morphological changes) of DT448/9PP on KMT2A-MLLT3 leukemia cells are more robust than the maximal effects of retinoid ligands such as all-trans retinoic acid and bexarotene. Although further delineation of mechanistic activity and cell-type susceptibility rules will be required, these data suggest that current small-molecule retinoids 
may incompletely activate the maturation pathways regulated by the retinoid receptors and that additional, unrealized potential may exist for retinoids in the treatment of forms of acute myeloid leukemia other than non-acute promyelocytic leukemia. In particular, myeloid maturation programs may be augmented by correctly activated retinoic $\mathrm{X}$ receptors and cells with monocytic potential may be susceptible to retinoid-induced maturation. An efficient, constitutively active $R X R A$ variant may enable further elucidation of this potential.

\section{Disclosures}

No conflicts of interest to disclose.

\section{Contributions}

$J S W, O d M$ and $M A F$ designed and performed experiments, and wrote the manuscript. GH, SS, AV, MPMG, and MR designed and performed experiments.

\section{Acknowledgments}

We thank the Alvin J. Siteman Cancer Center at Washington University School of Medicine and Barnes-Jewish Hospital in St. Louis, (MO, USA). for the use of the Flow Cytometry Core. The Siteman Cancer Center is supported in part by a National Cancer Institute Cancer Center Support grant (P30 CA91842). We thank Christopher Miller, Sai Ramakrishnan, Deborah Laflamme, Conner York, and Sangeetha Vadivelu for technical assistance.

\section{Funding}

This work was supported by National Institutes of Health grant R01 HL128447 (JSW), by the Siteman Investment Program (JSW), the Washington University SPORE DRP (JSW and MAF), the Children's Discovery Institute (JSW), the Alex's Lemonade Stand Foundation Young Investigator Award $(M A F)$, the National Institutes of Health $5 \mathrm{~K} 12 \mathrm{HD} 07622408(M A F)$, and grants from the Spanish Ministerio de Ciencia e Innovacion (MCI) (SAF201790604-REDT-NurCaMeIn, RTI2018-095928-BI00) (MR).

\section{References}

1. Oren T, Sher JA, Evans T. Hematopoiesis and retinoids: development and disease. Leuk Lymphoma. 2003;44(11):1881-1891.

2. Evans T. Regulation of hematopoiesis by retinoid signaling. Exp Hematol. 2005;33(9): 1055-1061.

3. Ricote M, Snyder CS, Leung HY, Chen J, Chien KR, Glass CK. Normal hematopoiesis after conditional targeting of RXRalpha in murine hematopoietic stem/progenitor cells. J Leukoc Biol. 2006;80(4):850-861.

4. Welch JS, Klco JM, Gao F, et al. Combination decitabine, arsenic trioxide, and ascorbic acid for the treatment of myelodysplastic syndrome and acute myeloid leukemia: a phase I study. Am J Hematol. 2011;86(9): 796-800.

5. Di Martino O, Niu H, Hadwiger G, et al. Endogenous and combination retinoids are active in myelomonocytic leukemias. Haematologica. 2021;106(4):1008-1021.

6. Martino OD, Welch JS. Retinoic acid receptors in acute myeloid leukemia therapy. Cancers. 2019;11(12):1915

7. Lubbert M, Grishina O, Schmoor C, et al. Valproate and retinoic acid in combination with decitabine in elderly nonfit patients with acute myeloid leukemia: results of a multicenter, randomized, $2 \times 2$, phase II trial. J Clin Oncol. 2020:38(3):257-270

8. vSchlenk RF, Frohling S, Hartmann F, et al. Phase III study of all-trans retinoic acid in previously untreated patients 61 years or older with acute myeloid leukemia. Leukemia. 2004;18(11):1798-1803.

9. McKeown MR, Corces MR, Eaton ML, et al. Super-enhancer analysis defines novel epigenomic subtypes of non-APL AML Including an RARalpha dependency targetable by SY1425 , a potent and selective RARalpha agonist. Cancer Discov. 2017;7(10):1136-1153.

10. Sakamoto K, Imamura $T$, Yano $M$, et al. Sensitivity of MLL-rearranged AML cells to all-trans retinoic acid is associated with the level of H3K4me2 in the RARalpha promoter region. Blood Cancer J. 2014;4(4):e205.

11. Niu H, Chacko J, Hadwiger G, Welch JS. Absence of natural intracellular retinoids in mouse bone marrow cells and implications for PML-RARA transformation. Blood Cancer J. 2015;5(2):e284.

12. Menendez-Gutierrez MP, Roszer T, Fuentes $\mathrm{L}$, et al. Retinoid X receptors orchestrate osteoclast differentiation and postnatal bone remodeling. J Clin Invest. 2015;125(2):809823.

13. Bray NL, Pimentel H, Melsted P, Pachter L. Near-optimal probabilistic RNA-seq quantification. Nat Biotechnol. 2016;34(5):525 527.

14. Ashburner M, Ball CA, Blake JA, et al. Gene ontology: tool for the unification of biology. The Gene Ontology Consortium. Nat Genet. 2000;25(1):25-29.

15. Subramanian A, Tamayo P, Mootha VK, et al. Gene set enrichment analysis: a knowledge-based approach for interpreting genome-wide expression profiles. Proc Natl Acad Sci U S A. 2005;102(43):15545-15550.

16. Ghosh JC, Yang X, Zhang A, et al. Interactions that determine the assembly of a retinoid X receptor/corepressor complex. Proc Natl Acad Sci U S A. 2002;99(9):58425847.

17. Lee WY, Noy N. Interactions of RXR with coactivators are differentially mediated by helix 11 of the receptor's ligand binding domain. Biochemistry. 2002;41(8):25002508.

18. Cordeiro TN, Sibille N, Germain P, et al Interplay of protein disorder in retinoic acid receptor heterodimer and its corepressor regulates gene expression. Structure. 2019;27(8):1270-1285.

19. Gao W, Liu J, Hu M, et al. Regulation of proteolytic cleavage of retinoid $\mathrm{X}$ receptoralpha by GSK-3beta. Carcinogenesis. 2013;34(6):1208-1215.

20. Fadel L, Reho B, Volko J, et al. Agonist binding directs dynamic competition among nuclear receptors for heterodimerization with retinoid X receptor. J Biol Chem. 2020;295(29):10045-10061

21. Devarakonda S, Gupta K, Chalmers MJ, et al. Disorder-to-order transition underlies the structural basis for the assembly of a transcriptionally active PGC-1alpha/ERRgamma complex. Proc Natl Acad Sci U S A. 2011;108(46):18678-18683.
22. Kersten S, Dong D, Lee W, Reczek PR, Noy $\mathrm{N}$. Auto-silencing by the retinoid X receptor. J Mol Biol. 1998;284(1):21-32.

23. Bourguet W, Vivat V, Wurtz JM, Chambon P, Gronemeyer H, Moras D. Crystal structure of a heterodimeric complex of RAR and RXR ligand-binding domains. Mol Cell. 2000;5(2):289-298.

24. Hiromori Y, Aoki A, Nishikawa J, Nagase H, Nakanishi T. Transactivation of the human retinoid $\mathrm{X}$ receptor by organotins: use of site-directed mutagenesis to identify critical amino acid residues for organotin-induced transactivation. Metallomics. 2015;7(7): 1180-1188

25. Halstead AM, Kapadia CD, Bolzenius J, et al. Bladder-cancer-associated mutations in RXRA activate peroxisome proliferator-activated receptors to drive urothelial proliferation. Elife. 2017;6:e30862.

26. Brown AL, Wilkinson CR, Waterman SR, et al. Genetic regulators of myelopoiesis and leukemic signaling identified by gene profiling and linear modeling. J Leukoc Biol. 2006;80(2):433-447.

27. Purton LE. Roles of retinoids and retinoic acid receptors in the regulation of hematopoietic stem cell self-renewal and differentiation. PPAR Res. 2007;2007:87934.

28. Mullen EM, Gu P, Cooney AJ. Nuclear receptors in regulation of mouse ES cell pluripotency and differentiation. PPAR Res. 2007;2007:61563.

29. Taschner S, Koesters C, Platzer B, et al. Down-regulation of RXRalpha expression is essential for neutrophil development from granulocyte/monocyte progenitors. Blood. 2007;109(3):971-979.

30. Yadav B, Pemovska T, Szwajda A, et al Quantitative scoring of differential drug sensitivity for individually optimized anticancer therapies. Sci Rep. 2014;4:5193.

31. Bianchi N, Ongaro F, Chiarabelli C, et al. Induction of erythroid differentiation of human K562 cells by cisplatin analogs. Biochem Pharmacol. 2000;60(1):31-40.

32. Niu $H$, Fujiwara $H$, di Martino $O$, et al Endogenous retinoid $\mathrm{X}$ receptor ligands in mouse hematopoietic cells. Sci Signal. 2017;10(503):eaan1011. 